\title{
The Impact of Coevolution and Abstention on the Emergence of Cooperation *
}

\author{
Marcos Cardinot, Colm O'Riordan, and Josephine Griffith \\ Dept. of Information Technology, National University of Ireland, Galway, Ireland \\ marcos.cardinot@nuigalway.ie
}

\begin{abstract}
This paper explores the Coevolutionary Optional Prisoner's Dilemma (COPD) game, which is a simple model to coevolve game strategy and link weights of agents playing the Optional Prisoner's Dilemma game. We consider a population of agents placed in a lattice grid with boundary conditions. A number of Monte Carlo simulations are performed to investigate the impacts of the COPD game on the emergence of cooperation. Results show that the coevolutionary rules enable cooperators to survive and even dominate, with the presence of abstainers in the population playing a key role in the protection of cooperators against exploitation from defectors. We observe that in adverse conditions such as when the initial population of abstainers is too scarce/abundant, or when the temptation to defect is very high, cooperation has no chance of emerging. However, when the simple coevolutionary rules are applied, cooperators flourish.
\end{abstract}

Keywords: Coevolution; Optional Prisoner's Dilemma Game; Evolutionary Game Theory; Cooperation.

\section{Introduction}

Evolutionary game theory in spatial environments has attracted much interest from researchers who seek to understand cooperative behaviour among rational individuals in complex environments. Many models have considered the scenarios where participant's interactions are constrained by particular graph topologies, such as lattices $[16,13]$, small-world graphs $[5,7]$, scale-free graphs $[18,20]$ and, bipartite graphs [9]. It has been shown that the spatial organisation of strategies on these topologies affects the evolution of cooperation [3].

The Prisoner's Dilemma (PD) game remains one of the most studied games in evolutionary game theory as it provides a simple and powerful framework to illustrate the conflicts inherent in the formation of cooperation. In addition, some extensions of the PD game, such as the Optional Prisoner's Dilemma (OPD) game, have been studied in an effort to investigate how levels of cooperation can be increased. In the OPD game, participants are afforded a third option -

* The final version will be available at Studies in Computational Intelligence (SCI), Springer, 2017. 
that of abstaining and not playing and thus obtaining the loner's payoff $(L)$. Incorporating this concept of abstention leads to a three-strategy game where participants can choose to cooperate, defect or abstain from a game interaction.

The vast majority of the spatial models in previous work have used static and unweighted networks. However, in many social scenarios that we wish to model, such as social networks and real biological networks, the number of individuals, their connections and environment are often dynamic. Thus, recent studies have also investigated the effects of evolutionary games played on dynamically weighted networks $[11,19,2,17,22]$ where it has been shown that the coevolution of both networks and game strategies can play a key role in resolving social dilemmas in a more realistic scenario.

In this paper we define and explore the Coevolutionary Optional Prisoner's Dilemma (COPD) game, which is a simple coevolutionary spatial model where both the game strategies and the link weights between agents evolve over time. In this model, the interaction between agents is described by an OPD game. Previous research on spatial games has shown that when the temptation to defect is high, defection is the dominant strategy in most cases. We believe that the combination of both optional games and coevolutionary rules can help in the emergence of cooperation in a wider range of scenarios.

Thus, given the Coevolutionary Optional Prisoner's Dilemma game (i.e., an OPD game in a spatial environment, where links between agents can be evolved), the aims of the work are to understand the effect of varying the parameters $T$ (temptation to defect), $L$ (loner's payoff), $\Delta$ and $\delta$ for both unbiased and biased environments.

By investigating the effect of these parameters, we aim to:

- Compare the outcomes of the COPD game with other games.

- Explore the impact of the link update rules and its properties.

- Investigate the evolution of cooperation when abstainers are present in the population.

- Investigate how many abstainers would be necessary to guarantee robust cooperation.

The results show that cooperation emerges even in extremely adverse scenarios where the temptation to defect is almost at its maximum. It can be observed that the presence of the abstainers are fundamental in protecting cooperators from invasion. In general, it is shown that, when the coevolutionary rules are used, cooperators do much better, being also able to dominate the whole population in many cases. Moreover, for some settings, we also observe interesting phenomena of cyclic competition between the three strategies, in which abstainers invade defectors, defectors invade cooperators and cooperators invade abstainers.

The paper outline is as follows: Section 2 presents a brief overview of the previous work in both spatial evolutionary game theory with dynamic networks and in the Optional Prisoner's Dilemma game. Section 3 gives an overview of the methodology employed, outlining the Optional Prisoner's Dilemma payoff matrix, the coevolutionary model used (Monte Carlo simulation), the strategy and link weight update rules, and the parameter values that are varied in order 
to explore the effect of coevolving both strategies and link weights. Section 4 discusses the benefits of combining the concept of abstention and coevolution. Section 5 further explore the effect of using the COPD game in an unbiased environment. Section 6 investigates the robustness of cooperative behaviour in a biased environment. Finally, Section 7 summarizes the main conclusions and outlines future work.

\section{Related Work}

The use of coevolutionary rules constitute a new trend in evolutionary game theory. These rules were first introduced by Zimmermann et al. [21], who proposed a model in which agents can adapt their neighbourhood during a dynamical evolution of game strategy and graph topology. Their model uses computer simulations to implement two rules: firstly, agents playing the Prisoner's Dilemma game update their strategy (cooperate or defect) by imitating the strategy of an agent in their neighbourhood with a higher payoff; and secondly, the network is updated by allowing defectors to break their connection with other defectors and replace the connection with a connection to a new neighbour selected randomly from the whole network. Results show that such an adaptation of the network is responsible for an increase in cooperation.

In fact, as stated by Perc and Szolnoki [15], the spatial coevolutionary game is a natural upgrade of the traditional spatial evolutionary game initially proposed by Nowak and May [13], who considered static and unweighted networks in which each individual can interact only with its immediate neighbours. In general, it has been shown that coevolving the spatial structure can promote the emergence of cooperation in many scenarios $[19,2]$, but the understanding of cooperative behaviour is still one of the central issues in evolutionary game theory.

Szolnoki and Perc [17] proposed a study of the impact of coevolutionary rules on the spatial version of three different games, i.e., the Prisoner's Dilemma, the Snow Drift and the Stag Hunt game. They introduce the concept of a teaching activity, which quantifies the ability of each agent to enforce its strategy on the opponent. It means that agents with higher teaching activity are more likely to reproduce than those with a low teaching activity. Differing from previous research [22,21], they also consider coevolution affecting either only the defectors or only the cooperators. They discuss that, in both cases and irrespective of the applied game, their coevolutionary model is much more beneficial to the cooperators than that of the traditional model.

Huang et al. [11] present a new model for the coevolution of game strategy and link weight. They consider a population of $100 \times 100$ agents arranged on a regular lattice network which is evolved through a Monte Carlo simulation. An agent's interaction is described by the classical Prisoner's Dilemma with a normalized payoff matrix. A new parameter, $\Delta / \delta$, is defined as the link weight amplitude and is calculated as the ratio of $\Delta / \delta$. They found that some values of $\Delta / \delta$ can provide the best environment for the evolution of cooperation. They 
also found that their coevolutionary model can promote cooperation efficiently even when the temptation of defection is high.

In addition to investigations of the classical Prisoner's Dilemma on spatial environments, some extensions of this game have also been explored as a means to favour the emergence of cooperative behaviour. For instance, the Optional Prisoner's Dilemma game, which introduces the concept of abstention, has been studied since Batali and Kitcher [1]. In their work, they proposed the opt-out or "loner's" strategy in which agents could choose to abstain from playing the game, as a third option, in order to avoid cooperating with known defectors. There have been a number of recent studies exploring this type of game $[20,8,14,12,10]$. Cardinot et al. [3] discuss that, with the introduction of abstainers, it is possible to observe new phenomena and, in a larger range of scenarios, cooperators can be robust to invasion by defectors and can dominate.

Although recent work has discussed the inclusion of optional games with coevolutionary rules [4], this still needs to be investigated in a wider range of scenarios. Therefore, our work aims to combine both of these trends in evolutionary game theory in order to identify favourable configurations for the emergence of cooperation in adverse scenarios, where, for example, the temptation to defect is very high or when the initial population of abstainers is either very scarce or very abundant.

\section{Methodology}

The goal of the experiments outlined in this section is to investigate the environmental settings when coevolution of both strategy and link weights of the Optional Prisoner's Dilemma on a weighted network takes place.

This section includes a complete description of the Optional Prisoner's Dilemma (PD) game, the spatial environment and the coevolutionary rules for both the strategy and link weights. Finally, we also outline the experimental set-up.

In the classical version of the Prisoner's Dilemma, two agents can choose either cooperation or defection. Hence, there are four payoffs associated with each pairwise interaction between the two agents. In consonance with common practice [11,13], payoffs are characterized by the reward for mutual cooperation $(R=1)$, punishment for mutual defection $(P=0)$, sucker's payoff $(S=0)$ and temptation to defect $(T=b$, where $1<b<2)$. Note that this parametrization refers to the weak version of the Prisoner's Dilemma game, where $P$ can be equal to $S$ without destroying the nature of the dilemma. In this way, the constraints $T>R>P \geq S$ maintain the dilemma.

The extended version of the PD game presented in this paper includes the concept of abstention, in which agents can not only cooperate $(C)$ or defect $(D)$ but can also choose to abstain $(A)$ from a game interaction, obtaining the loner's payoff $(L=l)$ which is awarded to both players if one or both abstain. As defined in other studies [3,16], abstainers receive a payoff greater than $P$ and less than $R$ (i.e., $P<L<R$ ). Thus, considering the normalized payoff matrix 
adopted, $0<l<1$. The payoff matrix and the associated values are illustrated in Table 1.

Table 1: The Optional Prisoner's Dilemma game matrix.

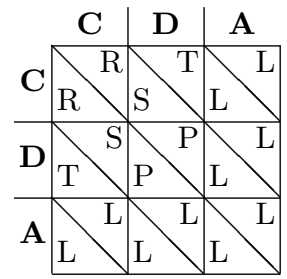

(a) Extended game matrix.

\begin{tabular}{l|c} 
Payoff & Value \\
\hline Temptation to defect (T) & ] $1,2[$ \\
Reward for mutual cooperation $(\mathrm{R})$ & 1 \\
Punishment for mutual defection $(\mathrm{P})$ & 0 \\
Sucker's payoff $(\mathrm{S})$ & 0 \\
Loner's payoff $(\mathrm{L})$ & ] $0,1[$
\end{tabular}

(b) Payoff values.

In these experiments, the following parameters are used: a $102 \times 102(N=$ $102^{2}$ ) regular lattice grid with periodic boundary conditions is created and fully populated with agents, which can play with their eight immediate neighbours (Moore neighbourhood). We adopt an unbiased environment (in which initially each agent is designated as a cooperator $(C)$, defector $(D)$ or abstainer $(A)$ with equal probability) as well as investigating a biased environment (in which the percentage of abstainers present in the environment is varied). Also, each edge linking agents has the same initial weight $w=1$, which will adaptively change in accordance with the interaction.

Monte Carlo methods are used to perform the Coevolutionary Optional Prisoner's Dilemma game. In one Monte Carlo (MC) step, each player is selected once on average. This means that one MC step comprises $N$ inner steps where the following calculations and updates occur:

- Select an agent $(x)$ at random from the population.

- Calculate the utility $u_{x y}$ of each interaction of $x$ with its eight neighbours (each neighbour represented as agent $y$ ) as follows:

$$
u_{x y}=w_{x y} P_{x y},
$$

where $w_{x y}$ is the edge weight between agents $x$ and $y$, and $P_{x y}$ corresponds to the payoff obtained by agent $x$ on playing the game with agent $y$.

- Calculate $U_{x}$ the accumulated utility of $x$, that is:

$$
U_{x}=\sum_{y \in \Omega_{x}} u_{x y},
$$

where $\Omega_{x}$ denotes the set of neighbours of the agent $x$. 
- In order to update the link weights, $w_{x y}$, between agents, compare the values of $u_{x y}$ and the average accumulated utility (i.e., $\bar{U}_{x}=U_{x} / 8$ ) as follows:

$$
w_{x y}=\left\{\begin{array}{ll}
w_{x y}+\Delta & \text { if } u_{x y}>\bar{U}_{x} \\
w_{x y}-\Delta & \text { if } u_{x y}<\bar{U}_{x} \\
w_{x y} & \text { otherwise }
\end{array},\right.
$$

where $\Delta$ is a constant such that $0 \leq \Delta \leq \delta$.

- In line with previous research [11,19], $w_{x y}$ is adjusted to be within the range

$$
1-\delta \leq w_{x y} \leq 1+\delta
$$

where $\delta(0<\delta \leq 1)$ defines the weight heterogeneity. Note that when $\Delta$ or $\delta$ are equal to 0 , the link weight keeps constant $(w=1)$, which results in the traditional scenario where only the strategies evolve.

- In order to update the strategy of $x$, the accumulated utility $U_{x}$ is recalculated (based on the new link weights) and compared with the accumulated utility of one randomly selected neighbour $\left(U_{y}\right)$. If $U_{y}>U_{x}$, agent $x$ will copy the strategy of agent $y$ with a probability proportional to the utility difference (Equation 5), otherwise, agent $x$ will keep its strategy for the next step.

$$
p\left(s_{x}=s_{y}\right)=\frac{U_{y}-U_{x}}{8(T-P)},
$$

where $T$ is the temptation to defect and $P$ is the punishment for mutual defection. This equation has been considered previously by Huang et al. [11].

Simulations are run for $10^{5} \mathrm{MC}$ steps and the fraction of cooperation is determined by calculating the average of the final $10^{3} \mathrm{MC}$ steps. To alleviate the effect of randomness in the approach, the final results are obtained by averaging 10 independent runs. The following scenarios are investigated:

- The benefits of coevolution and abstention.

- Presence of abstainers in the coevolutionary model.

- Inspecting the coevolutionary environment.

- Investigating the properties of the parameters $\Delta$ and $\delta$.

- Varying the number of states.

- Investigating the relationship between $\Delta / \delta, b$ and $l$.

- Investigating the robustness of cooperation in a biased environment.

\section{The Benefits of Coevolution and Abstention}

This section presents some of the main differences between the outcomes obtained by the proposed Coevolutionary Optional Prisoner's Dilemma (COPD) game and other models which do not adopt the concept of coevolution and/or abstention. In the COPD game, we also investigate how a population in an unbiased environment evolves over time. 


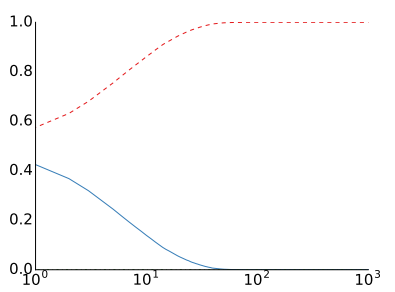

PD

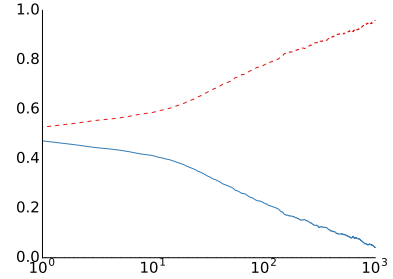

$\operatorname{CPD}(\Delta=0.72 ; \delta=0.8)$

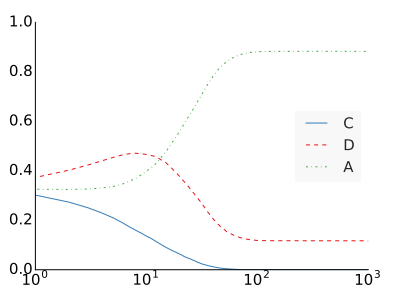

OPD $(l=0.6)$

Fig. 1: Comparison of the Prisoner's Dilemma (PD), the Coevolutionary Prisoner's Dilemma (CPD) and the Optional Prisoner's Dilemma (OPD) games. All with the same temptation to defect, $b=1.9$.

\subsection{Presence of Abstainers in the Coevolutionary Model}

In order to provide a means to effectively explore the impact of our coevolutionary model, i.e., the Coevolutionary Prisoner's Dilemma (COPD) game, in the emergence of cooperation, we start by investigating the performance of some of the existing models. Namely, the Coevolutionary Prisoner's Dilemma (CPD) game (i.e., same coevolutionary model as the COPD but without the concept of abstention), the traditional Prisoner's Dilemma (PD) game, and the Optional Prisoner's Dilemma game.

As shown in Figure 1, it can be observed that for both PD and CPD games, when the defector's payoff is very high (i.e., $b>1.7$ ) defectors spread quickly and dominate the environment. On the other hand, when abstainers are present in a static and unweighted network, i.e., playing the OPD game, we end up with abstainers dominating the environment. Undoubtedly, in many scenarios, having a population of abstainers is better than a population of defectors. However, it provides clear evidence that all these three models fail to sustain cooperation. In fact, results show that in this type of adverse environment (i.e., with a high temptation to defect), cooperation has no chance of emerging.

Surprisingly, as shown in Figure 2, when considering the Coevolutionary Optional Prisoner's Dilemma (COPD) game for the same environmental settings of Figure 1 (i.e., $l=0.6, \Delta=0.72$ and $\Delta=0.72$ ), with the temptation of defection almost at its peak (i.e., $b=1.9$ ), it is possible to reach high levels of cooperation.

Figure 2 shows a typical phase diagram for both CPD and COPD games for a fixed value of $\delta=0.8$ and $l=0.6$ (on the COPD game). It can be observed that if a given environmental setting (i.e, $b, \Delta$ and $\delta$ ) produces a stable population of cooperators in the CPD game, then the presence of abstainers will not change it. In other words, the COPD game does not affect the outcome of scenarios in which cooperation is stable in the absence of abstainers. Thus, the main changes occur in scenarios in which defection dominates or survives $(b>1.5)$.

To summarize, despite the fact that the Coevolutionary Prisoner's Dilemma (CPD) game succeeds in the promotion of cooperation in a wide range of scenar- 

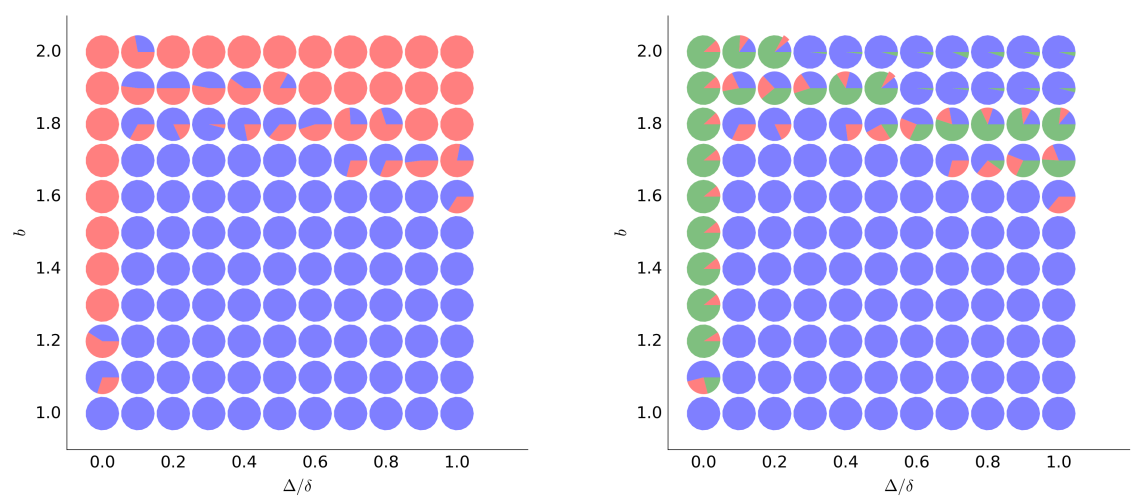

Fig. 2: Typical phase diagram for an initial balanced population playing the Coevolutionary Prisoner's Dilemma game (left) and the Coevolutionary Optional Prisoner's Dilemma game with $l=0.6$ (right), both with $\delta=0.8$.

ios, it is still not able to avoid the invasion by defectors in cases where $b>1.5$, which does not happen when the abstainers are present (i.e., COPD game).

\subsection{Inspecting the Coevolutionary Environment}

In order to further explain the results witnessed in the previous experiments, we investigate how the population evolves over time for the Coevolutionary Optional Prisoner's Dilemma game. Figure 3 features the time course of cooperation for three different values of $\Delta / \delta=\{0.0,0.2,1.0\}$, which are some of the critical points when $b=1.9, l=0.6$ and $\delta=0.8$. Based on these results, in Figure 4 we show snapshots for the Monte Carlo steps $0,45,1113$ and $10^{5}$ for the three scenarios shown in Figure 3.

We see from Figure 4 that for the traditional case (i.e., $\Delta / \delta=0.0$ ), abstainers spread quickly and reach a stable state in which single defectors are completely isolated by abstainers. In this way, as the payoffs obtained by a defector and an abstainer are the same, neither will ever change their strategy. In fact, even if a single cooperator survives up to this stage, for the same aforementioned reason, its strategy will not change either. In fact, the same behaviour is noticed for any value of $b>1.2$ and $\Delta / \delta=0$ (COPD in Figure 2).

When $\Delta / \delta=0.2$, it is possible to observe some sort of equilibrium between the three strategies. They reach a state of cyclic competition in which abstainers invade defectors, defectors invade cooperators and cooperators invade abstainers.

This behaviour, of balancing the three possible outcomes, is very common in nature where species with different reproductive strategies remain in equilibrium in the environment. For instance, the same scenario was observed as being responsible for preserving biodiversity in the neighbourhoods of the Escherichia coli, which is a bacteria commonly found in the lower intestine of warm-blooded organisms. According to Fisher [6], studies were performed with three natural 


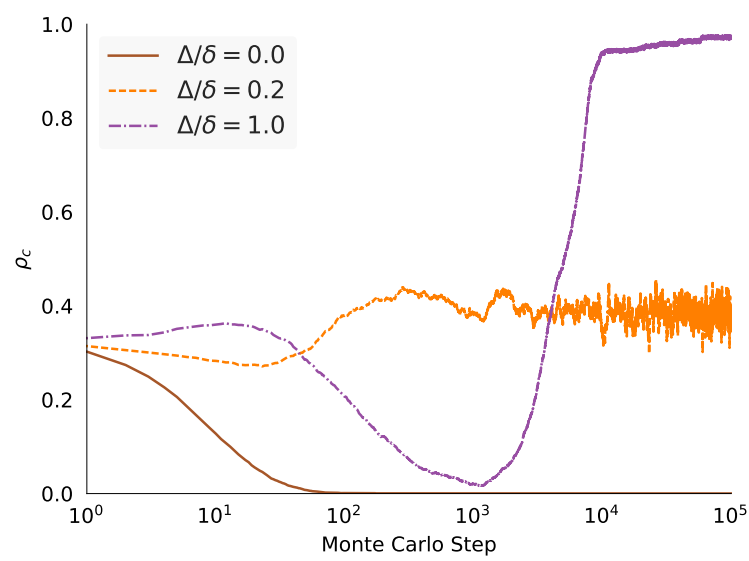

Fig. 3: Progress of the fraction of cooperation $\rho_{c}$ during a Monte Carlo simulation for $b=1.9, l=0.6$ and $\delta=0.8$.
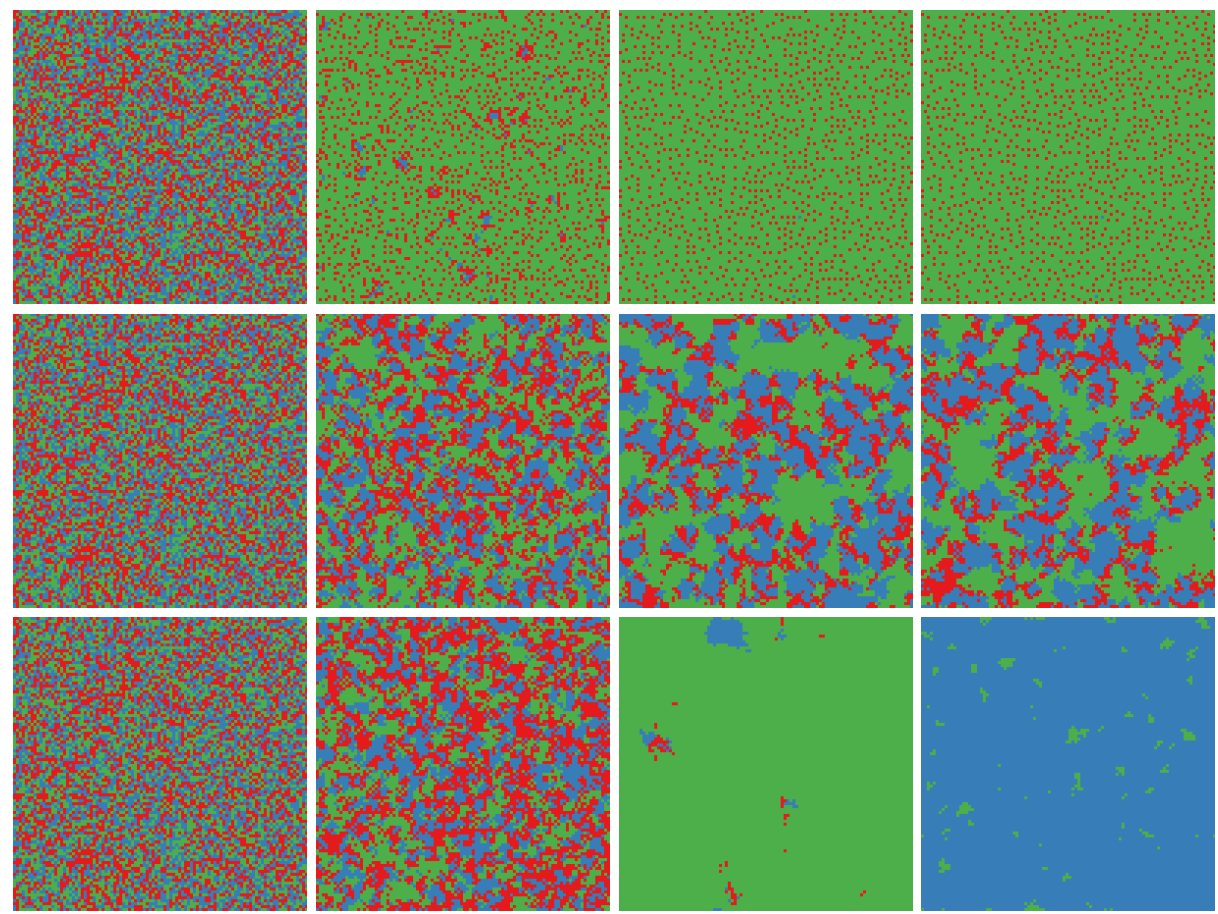

Fig. 4: Snapshots of the distribution of the strategy in the Monte Carlo steps 0, 45,1113 and $10^{5}$ (from left to right) for $\Delta / \delta$ equal to $0.0,0.2$ and 1.0 (from top to bottom). In this Figure, cooperators, defectors and abstainers are represented by the colours blue, red and green respectively. All results are obtained for $b=1.9$, $l=0.6$ and $\delta=0.8$. 
populations mixed together, in which one population produces a natural antibiotic but is immune to its effects; a second population is sensitive to the antibiotic but can grow faster than the third population; and the third population is resistant to the antibiotic.

Because of this balance, they observed that each population ends up establishing its own territory in the environment, as the first population could kill off any other bacteria sensitive to the antibiotic, the second population could use their faster growth rate to displace the bacteria which are resistant to the antibiotic, and the third population could use their immunity to displace the first population.

Another interesting behaviour is noticed for $\Delta / \delta=1.0$. In this scenario, defectors are dominated by abstainers, allowing a few clusters of cooperators to survive. As a result of the absence of defectors, cooperators invade abstainers and dominate the environment.

\section{Exploring the Coevolutionary Optional Prisoner's Dilemma game}

In this section, we present some of the relevant experimental results of the Monte Carlo simulations of the Coevolutionary Optional Prisoner's Dilemma game in an unbiased environment. That is, a well-mixed initial population with a balanced amount of cooperators, defectors and abstainers.

\subsection{Investigating the properties of $\Delta$ and $\delta$}

This section aims to investigate the properties of the presented model (Sect. 3) in regard to the parameters $\Delta$ and $\delta$. These parameters play a key role in the evolutionary dynamics of this model because they define the number of possible link weights that an agent is allowed to have (i.e., they define the number of states).

Despite the fact that the number of states is discrete, the act of counting them is not straightforward. For instance, when counting the number of states between $1-\delta$ and $1+\delta$ for $\Delta=0.2$ and $\delta=0.3$, we could incorrectly state that there are four possible states for this scenario (i.e., $\{0.7,0.9,1.1,1.3\}$ ). However, considering that the link weights of all edges are initially set to $w=1$, and due to the other constraints (Equations 3 and 4), the number of states is actually seven (i.e., $\{0.7,0.8,0.9,1.0,1.1,1.2,1.3\}$ ).

In order to better understand the relationship between $\Delta$ and $\delta$, we plot $\Delta, \delta$ and $\Delta / \delta$ as a function of the number of states (numerically counted) for a number of different values of both parameters (Figure 5). It was observed that given the pairs $\left(\Delta_{1}, \delta_{1}\right)$ and $\left(\Delta_{2} / \delta_{2}\right)$, if $\Delta_{1} / \delta_{1}$ is equal to $\Delta_{2} / \delta_{2}$, then the number of states of both settings is the same.

Figure 5 shows the ratio $\Delta / \delta$ as a function of the number of states. As we can see, although the function is non-linear and non-monotonic, in general, higher values of $\Delta / \delta$ have less states. 


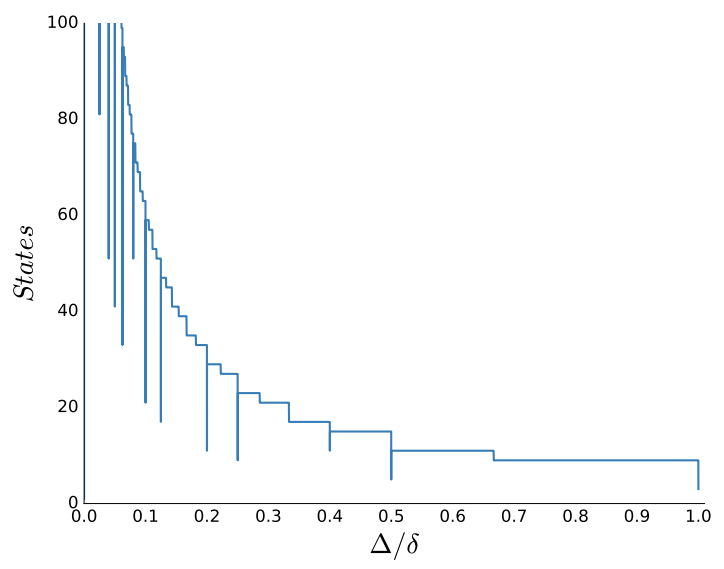

Fig. 5: The ratio $\Delta / \delta$ as a function of the number of states. For any combination of $\Delta$ and $\delta$, the ration $\Delta / \delta$ will always have the same number of states.

\subsection{Varying the Number of States}

Figure 6 shows the impact of the coevolutionary model on the emergence of cooperation when the ratio $\Delta / \delta$ varies for a range of fixed values of the loner's payoff $(l)$, temptation to defect $(b)$ and $\delta$. In this experiment, we observe that when $l=0.0$, the outcomes of the Coevolutionary Optional Prisoner's Dilemma (COPD) game are very similar to those observed by Huang et al. [11] for the Coevolutionary Prisoner's Dilemma (CPD) game. This result can be explained by the normalized payoff matrix adopted in this work (Table 1). Clearly, when $l=0.0$, there is no advantage in abstaining from playing the game, thus agents choose the option to cooperate or defect.

Results indicate that, in cases where the temptation to defect is very low (e.g, $b \leq 1.34$ ), the level of cooperation does not seem to be affected by the increment of the loner's payoff, except when the advantage of abstaining is very high (e.g, $l>0.8)$. However, these results highlight that the presence of the abstainers may protect cooperators from invasion. Moreover, the difference between the traditional Optional Prisoner's Dilemma (i.e., $\Delta / \delta=0.0$ ) for $l=\{0.0,0.6\}$ and all other values of $\Delta / \delta$ is strong evidence that our coevolutionary model is very advantageous to the promotion of cooperative behaviour.

Namely, when $l=0.6$, in the traditional case with a static and unweighted network $(\Delta / \delta=0.0)$, the cooperators have no chance of surviving; except, of course, when $b$ is very close to the reward for mutual cooperation $R$, where it is possible to observe scenarios of quasi-stable states of the three strategies or between cooperators and defectors. In fact, in the traditional OPD $(\Delta / \delta=0.0)$, when $l>0.0$ and $b>1.2$, abstainers are always the dominant strategy. However, when the coevolutionary rules are used, cooperators do much better, being also able to dominate the whole population in many cases. 

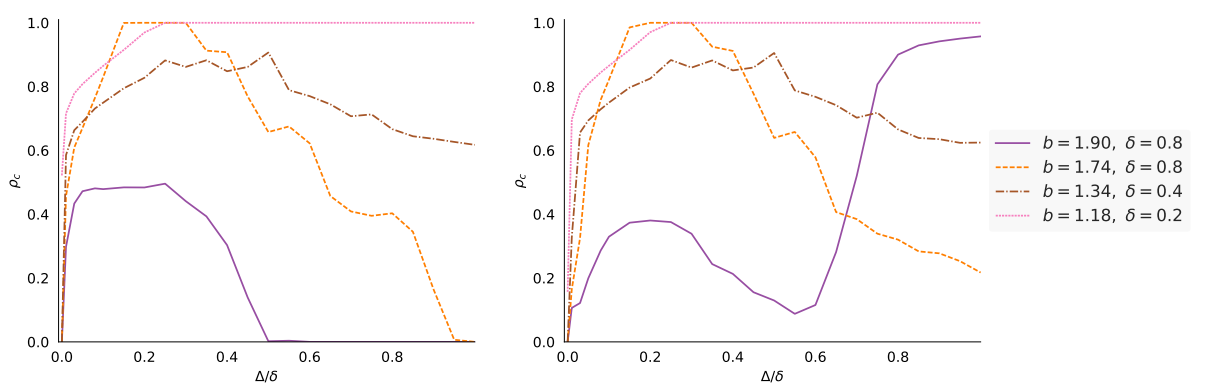

Fig. 6: Relationship between cooperation and the ratio $\Delta / \delta$ when the loner's payoff $(l)$ is equal to 0.0 (left) and 0.6 (right).

It is noteworthy that the curves in Figure 6 are usually non-linear and/or non-monotonic because of the properties of the ratio $\Delta / \delta$ in regard to the number of states of each combination of $\Delta$ and $\delta$ (Sect. 5.1).

\subsection{Investigating the Relationship between $\Delta / \delta, b$ and $l$}

To investigate the outcomes in other scenarios, we explore a wider range of settings by varying the values of the temptation to defect $(b)$, the loner's payoff $(l)$ and the ratio $\Delta / \delta$ for a fixed value of $\delta=0.8$.

As shown in Figure 7, cooperation is the dominant strategy in the majority of cases. Note that in the traditional case, with an unweighted and static network, i.e., $\Delta / \delta=0.0$, abstainers dominate in all scenarios illustrated in this ternary diagram. In addition, it is also possible to observe that certain combinations of $l$, $b$ and $\Delta / \delta$ guarantee higher levels of cooperation. In these scenarios, cooperators are protected by abstainers against exploitation from defectors.

Another observation is that defectors are attacked more efficiently by abstainers as we increase the loner's payoff $(l)$. Simulations reveal that, for any scenario, if the loner's payoff is greater than $0.7(l>0.7)$, defectors have no chance of surviving.

However, the drawback of increasing the value of $l$ is that it makes it difficult for cooperators to dominate abstainers, which might produce a quasi-stable population of cooperators and abstainers. It is noteworthy that it is a counterintuitive result from the COPD game, since the loner's payoff is always less than the reward for mutual cooperation (i.e., $L<R$ ), even for extremely high values of $L$. This scenario (population of cooperators and abstainers) should always lead cooperators to quickly dominate the environment.

In fact, it is still expected that, in the COPD game, cooperators dominate abstainers, but depending on the value of the loner's payoff, or the amount of abstainers in the population at this stage, it might take several Monte Carlo steps to reach a stable state, which is usually a state of cooperation fully dominating the population. 

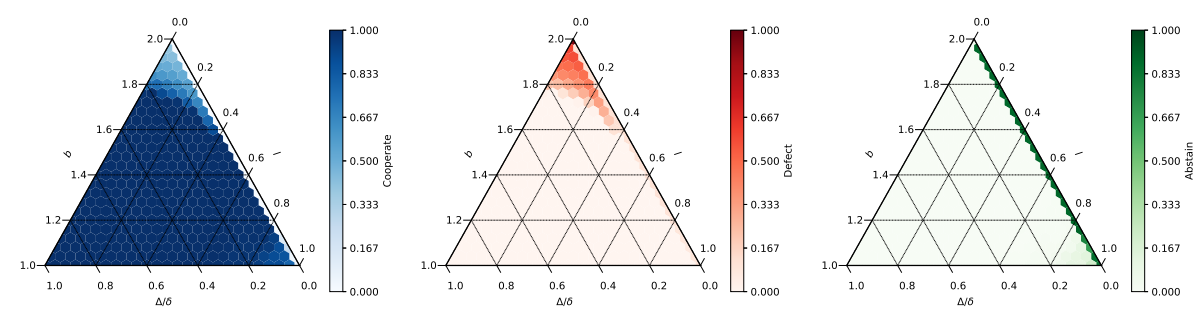

Fig. 7: Ternary diagrams of different values of $b, l$ and $\Delta / \delta$ for $\delta=0.8$.

An interesting behaviour is noticed when $l=[0.45,0.55]$ and $b>1.8$. In this scenario, abstainers quickly dominate the population, making a clear division between two states: before this range (defectors hardly die off) and after this range (defectors hardly survive). In this way, a loner's payoff value greater than $0.55(l>0.55)$ is usually the best choice to promote cooperation. This result is probably related to the difference between the possible utilities for each type of interaction, which still needs further investigation in future.

Although the combinations shown in Figure 7 for higher values of $\mathrm{b}(b>1.8)$ are just a small subset of an infinite number of possible values, it is clearly shown that a reasonable fraction of cooperators can survive even in an extremely adverse situation where the advantage of defecting is very high. Indeed, our results show that some combinations of high values of $l$ and $\delta$, such as for $\delta=0.8$ and $l=0.7$, can further improve the levels of cooperation, allowing for the full dominance of cooperation.

\section{Investigating the Robustness of Cooperation in a Biased Environment}

The previous experiments revealed that the presence of abstainers together with simple coevolutionary rules (i.e., the COPD game) act as a powerful mechanism to avoid the spread of defectors, which also allows the dominance of cooperation in a wide range of scenarios.

However, the distribution of the strategies in the initial population used in all of the previous experiments was uniform. That is, we have explored cases in which the initial population contained a balanced amount of cooperators, defectors and abstainers. Thus, in order to explore the robustness of these outcomes in regard to the initial amount of abstainers in the population, we now aim to investigate how many abstainers would be necessary to guarantee robust cooperation.

Figure 8 features the fraction of each strategy in the population (i.e., cooperators, defectors and abstainers) over time for fixed values of $b=1.9, \Delta=0.72$ and $\delta=0.8$. In this experiment, several independent simulations were performed, in which the loner's payoff $(l)$ and the number of abstainers in the initial population were varied from 0.0 to 1.0 and from $0.1 \%$ to $99.9 \%$, respectively. Other 
special cases were also analyzed, such as placing only one abstainer into a balanced population of cooperators and defectors, and placing only one defector and one cooperator in a population of abstainers. For the sake of simplicity, we report only the values of $l=\{0.2,0.6,0.8\}$ for an initial population with one abstainer and with 5\%,33\% and $90 \%$ abstainers, which are representative of the outcomes at other values also.

Note that, for all these simulations, the initial population of cooperators and defectors remained in balance. For instance, an initial population with $50 \%$ of abstainers, will consequently have $25 \%$ of cooperators and $25 \%$ of defectors.

Experiments reveal that the COPD game is actually extremely robust to radical changes in the initial population of abstainers. It has been shown that if the loner's payoff is greater than $0.55(l>0.55)$, then one abstainer might alone be enough to protect cooperators from the invasion of defectors (see Figures 8a, $8 \mathrm{~b}$ and $8 \mathrm{c}$ ). However, this outcome is only possible if the single abstainer is in the middle of a big cluster of defectors.

This outcome can happen because the payoff obtained by the abstainers is always greater than the one obtained by pairs of defectors (i.e., $L<P$ ). Thus, in a cluster of defectors, abstention is always the best choice. However, as this single abstainer reduces the population of defectors, which consequently increases the population of abstainers and cooperators in the population, defection may start to be a good option again due to the increase of cooperators. Therefore, the exploitation of defectors by abstainers must be as fast as possible, otherwise, they might not be able to effectively attack the population of defectors. In this scenario, the loner's payoff is the key parameter to control the speed in which abstainers invade defectors. This explains why a single abstainer is usually not enough to avoid the dominance of defectors when $l<0.55$.

In this way, as the loner's payoff is the only parameter that directly affects the evolutionary dynamics of the abstainers, intuition might lead one to expect to see a clear and perhaps linear relationship between the loner's payoff and the initial number of abstainers in the population. That is, given the same set of parameters, increasing the initial population of abstainers or the loner's payoff $(l)$ would probably make it easier for abstainers to increase or even dominate the population. Despite the fact that it might be true for high values of the loner's payoff (i.e., $l \geq 0.8$, as observed in Figure 8) is not applicable to other scenarios. Actually, as it is also shown in Figure 8, if the loner's payoff is less than 0.55, changing the initial population of abstainers does not change the outcome at all. When $0.55 \leq l<0.8$, a huge initial population of abstainers can actually promote cooperation best.

As discussed in Section 5.3, populations of cooperators and abstainers tend to converge to cooperation. In this way, the scenario showed in Figure 8 for $l=0.8$ will probably end up with cooperators dominating the population, but as the loner's payoff is close to the reward for mutual cooperation, the case in Figure 8i will converge faster than the one showed in Figure8l.

Another very counter-intuitive behaviour occurs in the range $l=[0.45,0.55]$ (this range may shift a little bit depending on the value of $b$ ), where the outcome 

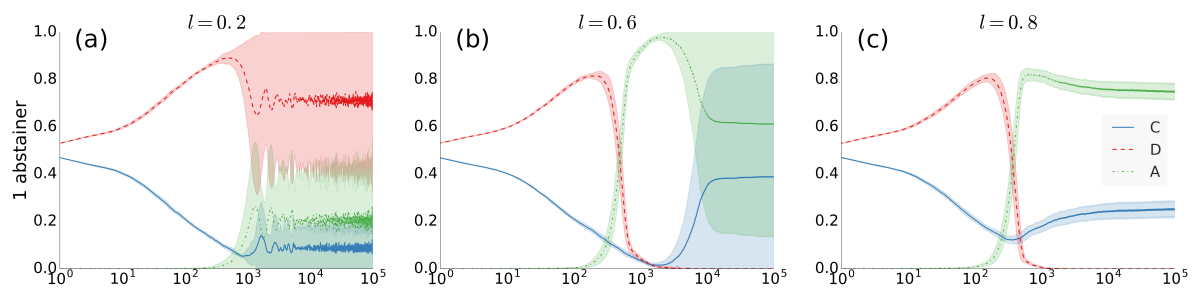

1.0 (d)
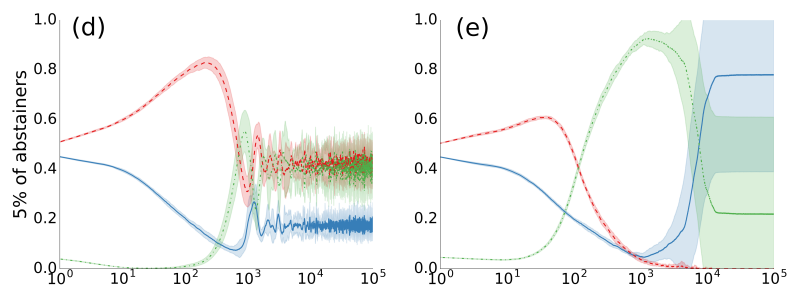

1.0 (f)

0.8

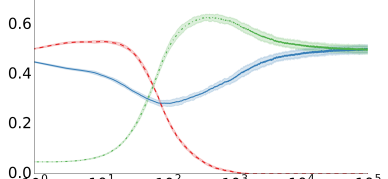

1.0 (g)
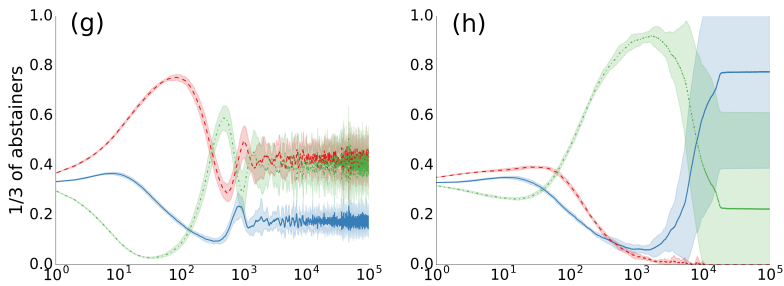

1.0 (i)

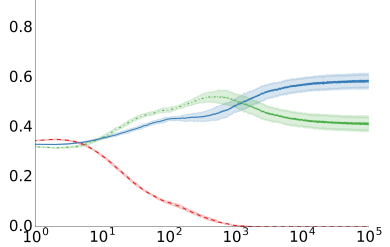

1.0 (j)
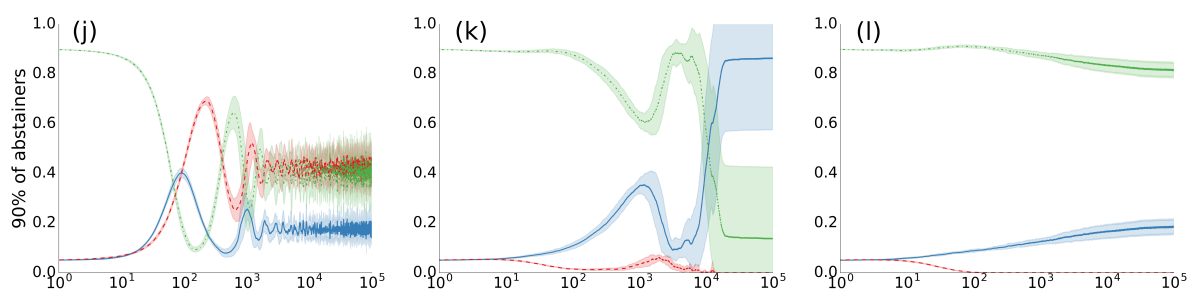

Fig. 8: Time course of each strategy for $b=1.9, \Delta=0.72, \delta=0.8$ and different values of $l$ (from left to right, $l=\{0.2,0.6,0.8\}$ ). The same settings are also tested on populations seeded with different amount of abstainers (i.e, from top to bottom: 1 abstainer, $5 \%$ of the population, $1 / 3$ of the population, $90 \%$ of the population). 
is usually of abstainers quickly dominating the population (Sect.5.3). In this scenario, we would expect that changes in the initial population of abstainers would at least change the speed in which the abstainers fixate in the population. That is, a huge initial population of abstainers would probably converge quickly. However, it was observed that the convergence speed is almost the same regardless of the size of the initial population of abstainers.

In summary, results show that an initial population with $5 \%$ of abstainers is usually enough to make reasonable changes in the outcome, increasing the chances of cooperators surviving or dominating the population.

\section{Conclusions and Future Work}

This paper studies the impact of a simple coevolutionary model in which not only the agents' strategies but also the network evolves over time. The model consists of placing agents playing the Optional Prisoner's Dilemma game in a dynamic spatial environment, which in turn, defines the Coevolutionary Optional Prisoner's Dilemma (COPD) game.

In summary, based on the results of several Monte Carlo simulations, it was shown that the COPD game allows for the emergence of cooperation in a wider range of scenarios than the Coevolutionary Prisoner's Dilemma (CPD) game (i.e., the same coevolutionary model in populations which do not have the option to abstain from playing the game). Results also showed that COPD performs much better than the traditional version of these games (i.e., the Prisoner's Dilemma (PD) and the Optional Prisoner's Dilemma (OPD) games) where only the strategies evolve over time in a static and unweighted network. Moreover, we observed that the COPD game is actually able to reproduce outcomes similar to other games by setting the parameters as follows:

- CPD: $l=0$.

- OPD: $\Delta=0($ or $\delta=0)$.

- PD: $l=0$ and $\Delta=0$ (or $\delta=0$ ).

Also, it was possible to observe that abstention acts as an important mechanism to avoid the dominance of defectors. For instance, in adverse scenarios such as when the defector's payoff is very high (i.e., $b>1.7$ ), for both PD and CPD games, defectors spread quickly and dominated the environment. On the other hand, when abstainers were present (COPD game), cooperation was able to survive and even dominate.

Furthermore, simulations showed that defectors die off when the loner's payoff is greater than $0.7(l>0.7)$. However, it was observed that increasing the loner's payoff makes it difficult for cooperators to dominate abstainers, which is a counter-intuitive result, since the loner's payoff is always less than the reward for mutual cooperation (i.e., $L<R$ ), this scenario should always lead cooperators to dominance very quickly. In this scenario, cooperation is still the dominant strategy in most cases, but it might require several Monte Carlo steps to reach a stable state. 
Results revealed that the COPD game also allows scenarios of cyclic dominance between the three strategies (i.e., cooperation, defection and abstention), indicating that, for some parameter settings, the COPD game is intransitive. That is, the population remains balanced in such a way that cooperators invade abstainers, abstainers invade defectors and defectors invade cooperators, closing a cycle.

We also explored the robustness of these outcomes in regard to the initial amount of abstainers in the population (biased population). In summary, it was shown that, in some of the scenarios, even one abstainer might alone be enough to protect cooperators from the invasion of defectors, which in turn increases the chances of cooperators surviving or dominating the population.

Although recent research has considered coevolving game strategy (with optional games) and link weights [4], this work presents a more complete analysis. We conclude that the combination of both of these trends in evolutionary game theory may shed additional light on gaining an in-depth understanding of the emergence of cooperative behaviour in real-world scenarios.

Future work will consider the exploration of different topologies and the influence of a wider range of scenarios, where, for example, agents could rewire their links, which, in turn, adds another level of complexity to the model. Future work will also involve applying our studies and results to realistic scenarios, such as social networks and real biological networks.

Acknowledgments. This work was supported by the National Council for Scientific and Technological Development (CNPq-Brazil).

\section{References}

1. Batali, J., Kitcher, P.: Evolution of altruism in optional and compulsory games. Journal of Theoretical Biology 175(2), 161-171 (1995)

2. Cao, L., Ohtsuki, H., Wang, B., Aihara, K.: Evolution of cooperation on adaptively weighted networks. Journal of Theoretical Biology 272(1), 8 - 15 (2011)

3. Cardinot, M., Gibbons, M., O'Riordan, C., Griffith, J.: Simulation of an optional strategy in the prisoner's dilemma in spatial and non-spatial environments. In: From Animals to Animats 14 (SAB 2016). pp. 145-156. Springer International Publishing, Cham (2016)

4. Cardinot, M., O'Riordan, C., Griffith, J.: The Optional Prisoner's Dilemma in a Spatial Environment: Coevolving Game Strategy and Link Weights. In: Proceedings of the 8th International Joint Conference on Computational Intelligence (IJCCI 2016). pp. 86-93 (2016)

5. Chen, X., Wang, L.: Promotion of cooperation induced by appropriate payoff aspirations in a small-world networked game. Physical Review E 77, 017103 (Jan 2008)

6. Fisher, L.: Rock, Paper, Scissors: Game Theory in Everyday Life. Basic Books (2008) 
7. Fu, F., Liu, L.H., Wang, L.: Evolutionary prisoner's dilemma on heterogeneous newman-watts small-world network. The European Physical Journal B 56(4), 367372 (2007)

8. Ghang, W., Nowak, M.A.: Indirect reciprocity with optional interactions. Journal of Theoretical Biology 365, 1-11 (2015)

9. Gómez-Gardeñes, J., Romance, M., Criado, R., Vilone, D., Sánchez, A.: Evolutionary games defined at the network mesoscale: The public goods game. Chaos 21(1) (2011)

10. Hauert, C., Traulsen, A., Brandt, H., Nowak, M.A.: Public goods with punishment and abstaining in finite and infinite populations. Biological Theory 3(2), 114-122 (2008)

11. Huang, K., Zheng, X., Li, Z., Yang, Y.: Understanding cooperative behavior based on the coevolution of game strategy and link weight. Scientific Reports 5, 14783 (2015)

12. Jeong, H.C., Oh, S.Y., Allen, B., Nowak, M.A.: Optional games on cycles and complete graphs. Journal of Theoretical Biology 356, 98-112 (2014)

13. Nowak, M.A., May, R.M.: Evolutionary games and spatial chaos. Nature 359(6398), 826-829 (1992)

14. Olejarz, J., Ghang, W., Nowak, M.A.: Indirect Reciprocity with Optional Interactions and Private Information. Games 6(4), 438-457 (2015)

15. Perc, M., Szolnoki, A.: Coevolutionary games - A mini review. Biosystems 99(2), 109-125 (2010)

16. Szabó, G., Hauert, C.: Evolutionary prisoner's dilemma games with voluntary participation. Physical Review E 66, 062903 (Dec 2002)

17. Szolnoki, A., Perc, M.: Promoting cooperation in social dilemmas via simple coevolutionary rules. The European Physical Journal B 67(3), 337-344 (2009)

18. Szolnoki, A., Perc, M.: Leaders should not be conformists in evolutionary social dilemmas. Scientific Reports 6, 23633 (2016)

19. Wang, Z., Szolnoki, A., Perc, M.: Self-organization towards optimally interdependent networks by means of coevolution. New Journal of Physics 16(3), 033041 (2014)

20. Xia, C.Y., Meloni, S., Perc, M., Moreno, Y.: Dynamic instability of cooperation due to diverse activity patterns in evolutionary social dilemmas. EPL 109(5), 58002 (2015)

21. Zimmermann, M.G., Eguíluz, V.M., San Miguel, M.: Cooperation, Adaptation and the Emergence of Leadership, pp. 73-86. Springer, Berlin, Heidelberg (2001)

22. Zimmermann, M.G., Eguíluz, V.M., San Miguel, M.: Coevolution of dynamical states and interactions in dynamic networks. Physical Review E 69, 065102 (2004) 